\title{
Toxocariasis: A Neglected Parasitic Disease with Public Health Importance
}

Oryan $\mathrm{A}^{*}$ and Alidadi S

Department of Pathology, School of Veterinary Medicine, Shiraz University, Shiraz, Iran

“Corresponding author: Oryan A, Department of Pathology, School of Veterinary Medicine, Shiraz University, Shiraz, Iran, Tel: +98 7132286950; Fax: +98 7132286940; E-mail: oryan@shirazu.ac.ir

Received date: June 25, 2015, Accepted date: June 27, 2015, Published date: June 29, 2015

Copyright: ( 2015 Oryan et al. This is an open-access article distributed under the terms of the Creative Commons Attribution License, which permits unrestricted use, distribution, and reproduction in any medium, provided the original author and source are credited.

\section{Editorial}

Toxocariasis together with Chagas disease, neurocysticercosis, toxoplasmosis and trichmoniasis are considered as the five most important neglected parasitic diseases [1]. Toxocariasis is a helminthic zoonosis mostly caused by two species of the ascarid nematodes Toxocara canis and to a lesser extent T. cati [2-4]. However, Toxocaris leonina belonging to the family Ascarididae can produce mixed infections with both Tocoxara species in dogs and cats [4]. In addition, it is able to infect humans; therefore it has zoonotic and public health importance $[4,5]$. Toxocaris leonina has been regarded as a causative agent of visceral larval migrans in human beings [5]. Toxocara canis and $T$. cati are common roundworms of the small intestine of canids and felids, respectively $[4,6]$. Toxocariasis carries a major health risk particularly in developing countries [7]. Human toxocariasis tends to be more prevalent in tropical and subtropical countries and rural populations than in temperate, urban and industrialized ones $[4,7,8]$ In tropical and subtropical regions, the humid climate provides surviving of parasite eggs in soil and this limits the control, preventive and eradicative programs [4]. Despite the poor hygiene and inappropriate anthelmintic treatments in dogs in rural settings, environmental contamination with Toxocara eggs is common in outdoor parks of urban and suburban settings $[4,8,9]$. Human beings as paratenic hosts become infected by ingestion of embryonated eggs in soil, from contaminated vegetables and fruits or consuming raw or undercooked meat and viscera of infected paratenic hosts including chicken, calves and lambs [7,10-12].

The causative agents are transmitted among canids and felids via a wide variety of routes including vertical, trans-placental (in dogs), trans-mammary and horizontal transmission [4]. Horizontal transmission occurs through ingestion of the embryonated eggs present in environment or ingestion of larvae in infected organs of paratenic hosts $[4,10]$. Distribution of toxocariasis is worldwide and is more prevalent in children $[13,14]$. Having a dog or cat as companion animal at home and close and frequent contact with such animals can predispose particularly children up to the age of 12 years to the ingestion of Toxocara eggs $[8,13,15]$. Poor hygiene, poverty and lack of education can exacerbate the exposure to Toxocara spp. [9]. Although toxocariasis has no racial predilection, males are usually more affected than females $[16,17]$.

Visceral and ocular involvement of the disease are called visceral larva migrans (VLM) or visceral toxocariasis and ocular larva migrans (OLM) or ocular toxocariasis, respectively $[1,3,18]$. Neural larva migrans (NLM) or neurological toxocariasis (NT) and convert or common toxocariasis (CT) are two less severe syndromes predominantly in adults and children, respectively $[3,4,8]$. The disease may vary from asymptomatic infection to severe lesions in different organs $[1,4,8,13]$. The symptoms and their severity depends on many factors such as the tissue invaded, number of migrating larvae, sites of larval migration, and age and immune status of the host $[3,8]$. In VLM that is mainly diagnosed in young children of less than 8 years of age, the clinical symptoms are usually associated to hepatic and pulmonary migration of the larvae $[3,4,10]$. Fever, abdominal pain, loss of appetite, vomiting, diarrhea, necrosis of the liver, hepatomegaly with splenomegaly or hepatosplenomegaly, coughing, wheezing, bronchospasm and asthma as well as pneumonia or bronchitis are symptoms related to patients with VLM [4,10]. Moreover, hypergamaglobinemia of immunoglobulin $\operatorname{IgG}$, IgM and $\operatorname{IgE}$ have been described in serological tests [10]. Similar to VLM, OLM mostly occurs in children [4]. In OLM, involvement is restricted to eyes and the optic nerves and the clinical symptoms are presented as unilateral impairment accompanying by strabismus $[1,8,19]$. Such clinical manifestations are initiated by an immune response to the larval migration into the eyes $[3,4]$.

The larvae are surrounded firstly by an eosinophilic abscess and eventually a granulomatous reaction $[4,19]$. Major consequences of OLM may firstly be blindness and secondary glaucoma [3,4]. Convert toxocariasis in children is characterized by fever, headache, anorexia, behavioral and sleep disturbances, cough, abdominal pain, hepatomegaly, nausea and vomiting [9]. Common toxocariasis in adults is associated with weakness, pruritus, rash, abdominal pain and pulmonary symptoms [4]. In cases of VLM, the larvae may disseminate via the bloodstream to anywhere in the host body such as the central nervous system (CNS) and occasionally peripheral nervous system and localize there; therefore causing neurological lesions and dysfunctions [16,20]. Involvement of the CNS leading to neurotoxocariasis is uncommon with few cases reported in the literature [3,21]. Migration of larvae of Toxocara spp. to the CNS in most instances is not associated with clinical central nervous signs However, rarely it may result in a variety of neurological disorders including meningo-encephalitis, myelitis, cerebral vasculitis, seizure and optic neuritis or combined presentations $[3,9,19,22]$. Pathological disorders such as demyelination, focal malacia and inflammatory cells infiltration especially eosinophils, lymphocytes, plasma cells and macrophages has also been described [23]. The cerebral lesions are mostly located in the white matter [3]. Following these manifestations, the patients complain about headache, weakness, confusion, epileptic seizure, neuropsychological disturbances, depression, sensory disturbances, reduced long and short term memory and probably cognitive and behavioral disorders [3,17,23]. Moreover, ataxia, dullness, rigor, tremor, paralysis, para-paresis, tetra-paresis, as well as urinary retention have been reported [3,23]. Toxocariasis has been suggested as an important risk factor for epilepsy, a chronic neurological disorder and therefore considerable attention has been directed toward the probable role of $T$. canis infection in epilepsy $[9,21]$. 
It seems the damages to the affected organs and CNS is due to the inflammatory reactions induced by the larvae $[16,24]$. The larvae produce glycosylated proteins inducing production of IL-5 and CD4Th2 response which eventually promote vascular adhesion and eosinophilic infiltration [16,25]. Eosinophilia is a common finding in blood and/or the cerebrospinal fluid (CSF) of toxocariasis cases $[16,22,26]$. It has been shown that in cerebral T. canis infections, the release of cytokines such as interleukin (IL) 5 and interferon $\gamma$ (IFN- $\gamma$ ) and inducible nitric oxide synthase (iNOS) is induced [22,24,26]. Various cells in the CNS including neurons, astrocytes, oligodendrocytes and microglia possess receptors of these cytokine $[24,26]$. The presence of IL-5 in the CNS can lead to the infiltration of eosinophils and subsequent degranulation of these cells results in the release of toxic mediators causing destruction of surrounding cells $[22,26]$. Intense release of cationic proteins from the eosinophil granules that are toxic to endothelial cells has been reported as a possible cause of these lesions [25]. It has been demonstrated that iNOS exerts deleterious effects on the murine brain during infection with $T$. canis $[24,26]$. On the other hand, the up-regulation of IFN- $\gamma$ in the CNS can result in activation of macrophages and microglia increasing nitric oxide production and subsequent tissue damage $[23,26]$.

However, $T$. canis in most instances is regarded as the main cause of human toxocariasis $[3,8]$, nonetheless, the risk of the disease caused by $T$. cati should not be underestimated or ignored. Therefore, the infection risk for humans with $T$. cati should be considered at least as high as that with $T$. canis so that cats are more likely to contaminate places like gardens due to their defecation habits [23].

Because the clinical signs and symptoms of toxocariasis are nonspecific, definitive diagnosis of the disease is a challenge. On the other hand, since poly-parasitism is common in tropical countries, diagnosis of VLM and CT cases is difficult. Generally, diagnosis of toxocariasis cases is based on history, clinical signs, various imaging techniques such as ultrasound, computed tomography (CT) and magnetic resonance imaging (MRI), biopsy and subsequent histopathological examination $[8,18]$. Beside serological techniques such as the indirect enzyme-linked immunosorbent assay (ELISA) on the basis of the excretory-secretory antigens of the third-stage larvae (L3) of $T$. canis, immunohistochemistry and molecular-based methods such as PCR can be helpful $[8,13,18]$. Although the diagnostic tests for VLM are immunological, they may not be reliable for OLM [10]. In OLM cases, ophthalmologic examination should be noted $[4,10]$. In the VLM cases, multiple oval hypoechoic lesions in the liver in sonographic examination and multifocal hepatic lesions present in the images from CT or MRI can be helpful in diagnosis [4]. Detection of the anti- $T$. canis antibodies in vitreous or aqueous humor is helpful in confirming early diagnosis of the OLM patients $[4,10]$. In addition, ocular ultrasound, fluorescein angiography, CT and optical coherence tomography can be useful [4]. Diagnosis of NT patients is usually based on high serum titers of Toxocara spp. antibodies, anti- T. canis antibodies in the CSF and eosinophilia in the CSF and/or blood, and close contact with dogs and cats $[4,22]$. In addition, the clinical and CT or MR imaging findings as well as the normalization of the CSF parameters during treatment can support and confirm the early diagnosis [16,22]. Multifocal, circumscribed granulomatous lesions in CNS with strong contrast enhancement or a combination of circumscribed and diffuse changes in chronic cases may be nonspecific MR imaging findings [4,22]. Angiography may confirm cerebral vasculitis in the NT cases [22]. In the NT cases, a list of diseases that can infect the CNS should be provided to make a differential diagnosis [4].

Severity and location of the lesions are important factors in selecting a suitable drug in toxocariasis treatment $[1,3,4]$. Albendazole is the choice for the treatment of toxocariasis in patients affected with VLM, CT or NT, and potent larvicides such as thiabendazole and diethylcarbamazine can also be effective in inhibiting larval migration $[3,4,8]$. Mebendazole is the second-line treatment for toxocariasis, whereas its gastrointestinal absorption is poor [10]. In addition, because the chronic inflammatory reactions by infiltration of eosinophils, lymphocytes, plasma cells and macrophages are more common than abscess formation in the CNS, corticosteroids are thought to be efficient as symptomatic treatment [19]. Treatment of the OLM cases is based on delicate ophthalmic procedures or even vitrectomy, anthelminthic chemotherapy and corticosteroids $[4,10]$.

Wide ranges of the definitive and paratenic hosts and multiplicity of the transmission routes of Toxocara species complicate the control, prevention and eradication of the parasite [4]. The high prevalence of the causative parasite in dogs and cats and also difficulties in preventing environmental contamination and human infection highlight its great importance as a zoonosis. Considering the high prevalence of toxocariasis in children, it is necessary to pay attention to the education of children and also the public. Proper cooking of foods can prevent the accidental ingestion of eggs. It should be noted that viable larvae of $T$. canis as well as $T$. cati have been found in meat even after prolonged periods of freezing $[7,23]$. Good hygiene practices should be encouraged and proper strategies should be designed and applied to prevent transmission of Toxocara spp.. Furthermore, probable relationship between toxocariasis and the global burden of epilepsy should be noted in establishing preventive measures. The importance of toxocariasis and persistent of the infective Toxocara larvae in meat should not be considered as neglected as previously thought. It should be added that veterinarians play an important role in combating the spread of Toxocara infection in places where a large number of dogs and cats are accompanied with pet owners. Regular stool examination and frequent chemotherapy of pets with albendazole or mebendazole can be effective in reducing the eggs number deposited in soil, therefore controlling the disease. In addition, control programs such as reducing the number of pet animals or limiting contacts of small children with them appear to be helpful. Control of the spread of feces of dogs and cats can help in controlling toxocariasis infection. Unfortunately, there is no vaccine available and an appropriate specific control program and development of proper vaccines against this zoonotic disease remain a challenge. Rapid identification and diagnosis of the larvae in paratenic hosts such as humans and development of an effective vaccine are essentials and basic requirement in designing any control program.

\section{References}

1. Moreira GM, Telmo Pde L, Mendonça M, Moreira AN, McBride AJ, et al. (2014) Human toxocariasis: current advances in diagnostics, treatment, and interventions. Trends Parasitol 30: 456-464.

2. Sajjadi SM, Oryan A, Jalali AR, Mehrabani D (1998) Study on the prevalence of Toxocara cati of stray cats in Shiraz, Iran. Parasitol Int 47 (Suppl.): 105-131, 128.

3. Strube C, Heuer L, Janecek E (2013) Toxocara spp. infections in paratenic hosts. Vet Parasitol 193: 375-389.

4. Macpherson CN (2013) The epidemiology and public health importance of toxocariasis: a zoonosis of global importance. Int J Parasitol 43: 999-1008. 
5. Mehrabani D, Sadjjadi SM, Oryan A (2002) Prevalence of gastrointestinal nematode parasites in stray dogs in Shiraz, southern Iran. J Appl Anim Res 22: 157-160.

6. Mehrabani D, Sadjjadi SM, Oryan A, Tabatabase SMR (1998) Nematode parasites of stray dogs in Shiraz, southern Iran. Parasitol Int 47 (Suppl.): 133-281, 162.

7. Oryan A, Sadjjadi SM, Azizi S (2010) Longevity of Toxocara cati larvae and pathology in tissues of experimentally infected chickens. Korean J Parasitol 48: 79-80.

8. Rubinsky-Elefant G, Hirata CE, Yamamoto JH, Ferreira MU (2010) Human toxocariasis: diagnosis, worldwide seroprevalences and clinical expression of the systemic and ocular forms. Ann Trop Med Parasitol 104: 3-23.

9. Quattrocchi G, Nicoletti A, Marin B, Bruno E, Druet-Cabanac M, et al. (2012) Toxocariasis and epilepsy: systematic review and meta-analysis. PLoS Negl Trop Dis 6: e1775.

10. Despommier D (2003) Toxocariasis: clinical aspects, epidemiology, medical ecology, and molecular aspects. Clin Microbiol Rev 16: 265-272.

11. Azizi S, Oryan A, Sadjjadi SM, Zibaei M (2007) Histopathologic changes and larval recovery of Toxocara cati in experimentally infected chickens. Parasitol Res 102: 47-52.

12. Zibaei M, Sadjjadi SM, Sarkari B, Oryan A, Uga S (2009) In vitro cultivation of Toxocara cati adult worms for production of eggs and evaluation of oviposition. Helminthologia 46: 28-30.

13. Sadjjadi SM, Khosravi M, Mehrabani D, Orya A (2000) Seroprevalence of toxocara infection in school children in Shiraz, southern Iran. J Trop Pediatr 46: 327-330.

14. Sadjjadi SM, Oryan A, Jalali AR, Mehrabani D (2001) Prevalence and intensity of infestation with Toxocara cati in stray cats in Shiraz, Iran. Vet Arhiv 71: 149-157.

15. Fu CJ, Chuang TW, Lin HS, Wu CH, Liu YC, et al. (2014) Seroepidemiology of Toxocara canis infection among primary schoolchildren in the capital area of the Republic of the Marshall Islands. BMC Infect Dis 14: 261
16. Salvador S, Ribeiro R, Winckler MI, Ohlweiler L, Riesgo R (2010) Pediatric neurotoxocariasis with concomitant cerebral, cerebellar, and peripheral nervous system involvement: case report and review of the literature. J Pediatr (Rio J) 86: 531-534.

17. Fan CK, Liao CW, Cheng YC (2013) Factors affecting disease manifestation of toxocarosis in humans: genetics and environment. Vet Parasitol 193: 342-352.

18. Zibaei M, Sadjjadi SM, Karamian M, Uga S, Oryan A, et al. (2013) A comparative histopathology, serology and molecular study, on experimental ocular toxocariasis by Toxocara cati in Mongolian gerbils and Wistar rats. Biomed Res Int 2013: 109580.

19. Helsen G, Vandecasteele SJ, Vanopdenbosch LJ (2011) Toxocariasis presenting as encephalomyelitis. Case Rep Med 2011: 503913.

20. Oryan A, Sadjjani SM, Azizi S (2009) The effects of benzimidazoles on the larval stage of Toxocara cati in experimentally infected chickens. Trop Biomed 26: 30-39.

21. El-Tantawy NL, El-Nahas HA, Salem DA-B, Salem NA-B, Hasaneen BM (2013) Seroprevalence of Toxoplasma gondii and Toxocara Spp in children with cryptogenic epilepsy. AJIDM 1: 92-95.

22. Xinou E, Lefkopoulos A, Gelagoti M, Drevelegas A, Diakou A, et al. (2003) $\mathrm{CT}$ and MR imaging findings in cerebral toxocaral disease. AJNR Am J Neuroradiol 24: 714-718.

23. Janecek E, Beineke A, Schnieder T, Strube C1 (2014) Neurotoxocarosis: marked preference of Toxocara canis for the cerebrum and T. cati for the cerebellum in the paratenic model host mouse. Parasit Vectors 7: 194.

24. Holland CV, Hamilton CM (2013) The significance of cerebral toxocariasis: a model system for exploring the link between brain involvement, behaviour and the immune response. J Exp Biol 216: 78-83.

25. Fellrath JM, Magnaval JF2 (2014) Toxocariasis after slug ingestion characterized by severe neurologic, ocular, and pulmonary involvement. Open Forum Infect Dis 1: ofu063.

26. Hamilton CM, Brandes S, Holland CV, Pinelli E (2008) Cytokine expression in the brains of Toxocara canis-infected mice. Parasite Immunol 30: 181-185. 